\title{
Automated Mutual Explicit Induction Proof in Separation Logic
}

\author{
Quang-Trung Ta Ton Chanh Le Siau-Cheng Khoo Wei-Ngan Chin \\ \{taqt, chanhle, khoosc, chinwn\}@comp.nus.edu.sg \\ School of Computing, National University of Singapore
}

\begin{abstract}
We present a sequent-based deductive system for automatically proving entailments in separation logic by using mathematical induction. Our technique, called mutual explicit induction proof, is an instance of Noetherian induction. Specifically, we propose a novel induction principle on a well-founded relation of separation logic model, and follow the explicit induction methods to implement this principle as inference rules, so that it can be easily integrated into a deductive system. We also support mutual induction, a natural feature of implicit induction, where the goal entailment and other entailments derived during the proof search can be used as hypotheses to prove each other. We have implemented a prototype prover and evaluated it on a benchmark of handcrafted entailments as well as benchmarks from a separation logic competition.
\end{abstract}

\section{Introduction}

Separation logic (SL) $[21,29]$ has been actively used recently to reason about imperative programs that alter data structures. For example, the static analysis tool Infer [1] of Facebook has been using SL to discover critical memory safety bugs in Android and iOS applications. One of the pivotal features making the success of SL is the separating conjunction operator $(*)$, which is used to describe the separation of computer memory. In particular, the assertion $p * q$ denotes a memory portion which can be decomposed into two disjoint sub-portions held by $p$ and $q$, respectively. In addition, SL is also equipped with the ability for users to define inductive heap predicates $[28,5,15]$. The combination of the separating conjunction and inductive heap predicates makes SL expressive enough to model various types of recursive data structures, such as linked lists and trees.

However, this powerful expressiveness also poses challenges in reasoning about SL entailments. Considerable researches have been conducted on the SL entailment proving problem, including the works $[5,7,11]$ related to mathematical induction. In particular, Brotherston et al. [5,7] propose the cyclic proof, which allows proof trees to contain cycles, and can be perceived as infinite derivation trees. Furthermore, during the proof derivation, induction hypotheses are not explicitly identified via applications of induction rules; instead, they are implicitly obtained via the discovery of valid cycle proofs. Consequently, a soundness condition needs to be checked globally on proof trees. On the other hand, Chu et al. [11] apply structural induction on inductive heap predicates for proving 
SL entailments. During proof search, this technique dynamically uses derived entailments as induction hypotheses. When applying induction hypotheses, it performs a local check to ensure that predicates in the target entailments are substructures of predicates in the entailments captured as hypotheses. This dynamicity in hypothesis generation enables multiple induction hypotheses within a single proof path to be exploited; however, it does not admit hypotheses obtained from different proof paths.

In this work, we develop a sequent-based deductive system for proving SL entailments by using mathematical induction. Our technique is an instance of Noetherian induction [8], where we propose a novel induction principle based on a wellfounded relation of SL models. Generally, proof techniques based on Noetherian induction are often classified into two categories, i.e., explicit and implicit induction [8], and each of them presents advantages over the other. We follow the explicit induction methods to implement the induction principle as inference rules, so that it can be easily integrated into a deductive system, and the soundness condition can be checked locally in each application of inference rules. In addition, since the well-founded relation defined in our induction principle does not depend directly on the substructure relationship, induction hypotheses gathered in one proof path can be used for hypothesis applications at other proof paths of the entire proof tree. Thus, our induction principle also favors mutual induction, a natural feature of implicit induction, in which the goal entailment and other entailments derived during the proof search can be used as hypotheses to prove each other. Our proof technique, therefore, does not restrict induction hypotheses to be collected from only one proof path, but rather from all derived paths of the proof tree.

Related work. The entailment proving problem in SL has been actively studied recently. Various sound and complete techniques have been introduced, but they deal with only pre-defined inductive heap predicates, whose definitions and semantics are given in advance $[2,3,12,23,22,4,24,25]$. Since these techniques are designated to only certain classes of pre-defined predicates, they are not suitable for handling general inductive heap predicates.

Iosif et al. [15,16] and Enea et al. [13] aim to prove entailments in more general SL fragments by translating SL assertions into tree automata. However, these approaches still have certain restrictions on inductive heap predicates, such as the predicates must have the bounded tree width property, or they are variants of linked list structures. Proof techniques proposed by Nguyen et al. $[20,19,10]$ and by Madhusudan et al. [26] can prove SL entailments with general inductive heap predicates. Nonetheless, these techniques are semi-automated since users are required to provide supplementing lemmas to assist in handling those predicates. In [14], Enea et al. develop a mechanism to automatically synthesize these supporting lemmas, but solely limited to certain kinds of lemmas, i.e., composition lemmas, completion lemmas and stronger lemmas. 
Cyclic proof $[5,7]$ and induction proof in [11] are most closely related to our approach. We recall the aforementioned comments that cyclic proof requires soundness condition to be checked globally on proof trees, whereas proof technique in [11] restricts that induction hypotheses collected from one path of proof tree cannot be used to prove entailments in other paths. Our work differs from them as we not only allow soundness condition to be checked locally at inference rule level, but also support mutual induction where entailments from different proof paths can be used as hypotheses to prove each other.

Contribution. Our contributions in this work are summarized as follows:

- We define a well-founded relation on SL models and use it to construct a novel mutual induction principle for proving SL entailments.

- We develop a deductive system for proving SL entailments based on the proposed mutual induction principle, and prove soundness of the proof system.

- We implement a prototype prover, named Songbird, and experiment on it with benchmarks of handcrafted entailments as well as entailments collected from SL-COMP, an SL competition. Our prover is available for both online use and download at: http://loris-5.d2.comp.nus.edu.sg/songbird/.

\section{Motivating Example}

We consider the procedure traverse in Fig. 1, which traverses a linked list in an unusual way, by randomly jumping either one or two steps at a time. In order to verify memory safety of this program, automated verification tools such as $[9,17]$ will first formulate the shape of the computer memory manipulated by traverse. Suppose the initially discovered shape is represented by an inductive heap predicate $\operatorname{tmp}(x)$ in $\mathrm{SL}$, defined as:

$$
\operatorname{tmp}(x) \triangleq \operatorname{emp} \vee \exists u .(x \mapsto u * \operatorname{tmp}(u)) \vee \exists u, v \cdot(x \mapsto u * u \mapsto v * \operatorname{tmp}(v))
$$

Intuitively, $\operatorname{tmp}(x)$ covers three possible cases of the shape, which can be an empty memory emp (when $\mathrm{x}==$ NULL), or be recursively expanded by a single data structure $x \mapsto u$ (when traverse jumps one step), or be recursively expanded by two structures $x \mapsto u$ and $u \mapsto v$ (when traverse jumps two steps). Note that $x \mapsto u$ and $u \mapsto v$ are SL predicates modeling the data structure node. Details about the SL syntax will

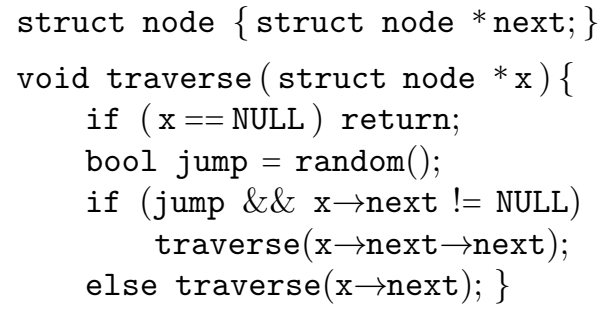

Fig. 1: A linked-list traversal algorithm with random jump be explained in Section 3 .

Since the derived shape is anomalous, the verifiers or users may want to examine if it is actually a linked list segment, modeled by the following predicate:

$$
\mathrm{I} \mathbf{s}(x, y) \triangleq(\operatorname{emp} \wedge x=y) \vee \exists w \cdot(x \mapsto w * \operatorname{ls}(w, y))
$$


This can be done by checking the validity of the following entailment:

$$
E \triangleq \operatorname{tmp}(x) \vdash \exists y \cdot \operatorname{ls}(x, y)
$$

In the semantics of SL, the entailment $E$ is said to be valid, if all memory models satisfying $\operatorname{tmp}(x)$ also satisfy $\exists y \operatorname{ls}(x, y)$. To prove it by induction, $E$ is firstly recorded as an induction hypothesis $(\mathrm{IH})$, then the predicate $\operatorname{tmp}(x)$ is analyzed in each case of its definition, via a method called unfolding, to derive new entailments $E_{1}, E_{2}, E_{3}$ as follows.

$$
\begin{gathered}
E_{1} \triangleq \mathrm{emp} \vdash \exists y \cdot \operatorname{ls}(x, y) \quad E_{2} \triangleq x \mapsto u * \operatorname{tmp}(u) \vdash \exists y \cdot \operatorname{ls}(x, y) \\
E_{3} \triangleq x \mapsto u * u \mapsto v * \operatorname{tmp}(v) \vdash \exists y \cdot \operatorname{ls}(x, y)
\end{gathered}
$$

The entailment $E_{1}$ can be easily proved by unfolding the predicate $\operatorname{ls}(x, y)$ in the right side by its base case to obtain a valid entailment emp $\vdash \exists y$. $(\mathrm{emp} \wedge x=y)$. On the contrary, the entailment $E_{2}$ can only be proved by using the induction hypothesis $E$. Its (simplified) proof tree can be depicted in Fig. 2.

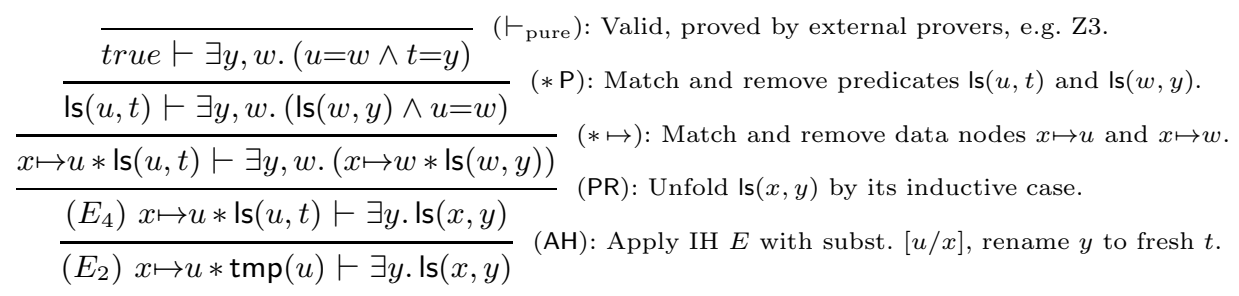

Fig. 2: Proof tree of $E_{2}$, using induction hypothesis $E$

We can also prove $E_{3}$ by the same method, i.e., applying the IH $E$, and its proof tree is shown in Fig. 3.

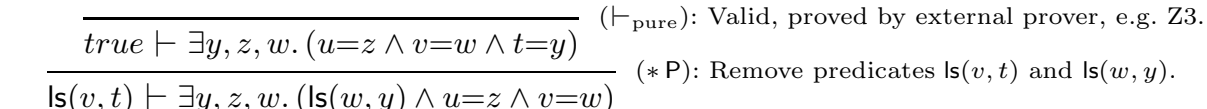

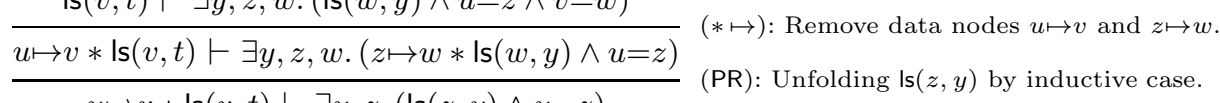

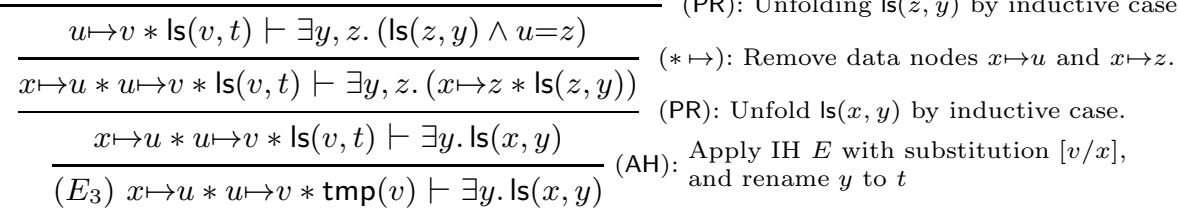

Fig. 3: Ordinary proof tree of $E_{3}$, using induction hypothesis $E$

Using a different strategy, we observe that once $E_{2}$ is proved, entailments derived during its proof, i.e., $E_{2}$ and $E_{4}$, can be used as hypotheses to prove $E_{3}$. In this case, the new proof of $E_{3}$ is much simpler than the above original induction proof, as demonstrated in Fig. 4; the proving process, therefore, is more efficient.

In the new proof tree, the entailment $E_{4}$ can be directly used as a hypothesis to prove other entailments since it is already proven valid (see Fig. 2). However, 
when $E_{2}$ is applied to prove $E_{3}$, thus prove $E$, it is not straightforward to conclude about $E$, since the validity of $E_{2}$ is still unknown. This is because the proof of $E_{2}$ in Fig. 2 also uses $E$ as a hypothesis. Therefore, $E$ and $E_{2}$ jointly form a mutual induction proof, in which they can be used to prove each other. The theoretical principle of this proof technique will be introduced in Section 4 .

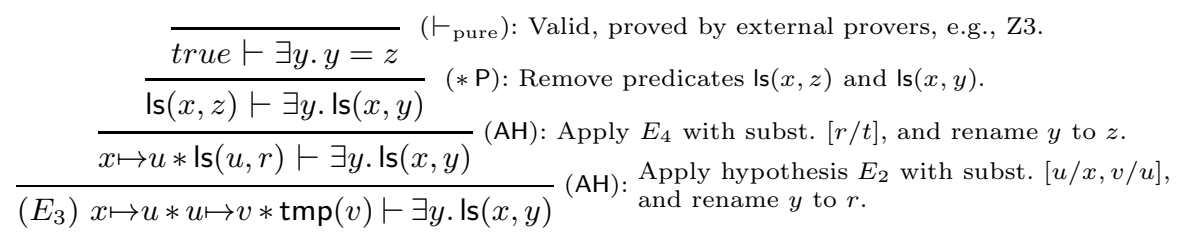

Fig. 4: New proof tree of $E_{3}$, using hypotheses $E_{2}$ and $E_{4}$

\section{Theoretical background}

In this work, we consider the symbolic-heap fragment of separation logic with arbitrary user-defined inductive heap predicates. We denote this logic fragment as $\mathrm{SL}_{\mathrm{ID}}$. It is similar to those introduced in $[15,6]$, but extended with linear arithmetic (LA) to describe more expressive properties of the data structures, such as size or sortedness. The syntax and semantics of the SLID assertions and their entailments are introduced in this section.

\subsection{Symbolic-heap Separation Logic}

Syntax. The syntax of our considered separation logic fragment $\mathrm{SL}_{\mathrm{ID}}$ is described in Fig. 5. In particular, the predicate emp represents an empty memory. The singleton heap predicate $x \stackrel{\iota}{\mapsto} x_{1}, \ldots, x_{n}$ models an $n$-field single data structure in memory where $x$ points-to; its data type is represented by a unique sort $\iota^{1}$ and values of its fields are captured by $x_{1}, \ldots, x_{n}$. The inductive heap predicate $\mathrm{P}\left(x_{1}, \ldots, x_{n}\right)$ models a recursively defined data structure, which is formally defined in Definition 1. These three heap predicates, called spatial atoms, compose the spatial assertions $\Sigma$ via the separating conjunction operator $*$. $\Pi$ denotes pure assertions in linear arithmetic, which do not contain any spatial atoms.

Definition 1 (Inductive heap predicate). A system of $k$ inductive heap predicates $\mathrm{P}_{i}$ of arity $n_{i}$ and parameters $x_{1}^{i}, \ldots, x_{n_{i}}^{i}$, with $i=1, \ldots, k$, are syntactically defined as follows:

$$
\left\{\mathrm{P}_{i}\left(x_{1}^{i}, \ldots, x_{n_{i}}^{i}\right) \triangleq F_{1}^{i}\left(x_{1}^{i}, \ldots, x_{n_{i}}^{i}\right) \vee \cdots \vee F_{m_{i}}^{i}\left(x_{1}^{i}, \ldots, x_{n_{i}}^{i}\right)\right\}_{i=1}^{k}
$$

where $F_{j}^{i}\left(x_{1}^{i}, \ldots, x_{n_{i}}^{i}\right)$, with $1 \leq j \leq m_{i}$, is a definition case of $\mathrm{P}_{i}\left(x_{1}^{i}, \ldots, x_{n_{i}}^{i}\right)$. Moreover, $F_{j}^{i}$ is a base case of $\mathrm{P}_{i}$, if it does not contain any predicate symbol which is (mutually) recursively defined with $\mathrm{P}_{i}$; otherwise, it is an inductive case.

\footnotetext{
${ }^{1}$ Note that for the simplicity of presenting the motivating example, we have removed the sort $\iota$ from the SL singleton heap predicate denoting the data structure node.
} 
$c, x, \iota, \mathrm{P}$ resp. denote constants, variables, data sorts, and predicate symbols.

$$
\begin{array}{rlrl}
e::= & c|x|-e\left|e_{1}+e_{2}\right| e_{1}-e_{2} & \text { Integer expressions } \\
a::= & \text { nil } \mid x & & \text { Spatial expressions } \\
\Pi::= & a_{1}=a_{2}\left|a_{1} \neq a_{2}\right| e_{1}=e_{2}\left|e_{1} \neq e_{2}\right| & & \text { Pure assertions } \\
& e_{1}>e_{2}\left|e_{1} \geq e_{2}\right| e_{1}<e_{2}\left|e_{1} \leq e_{2}\right| & \\
& \neg \Pi\left|\Pi_{1} \wedge \Pi_{2}\right| \Pi_{1} \vee \Pi_{2}\left|\Pi_{1} \Rightarrow \Pi_{2}\right| \forall x . \Pi \mid \exists x . \Pi & \\
\Sigma::= & \operatorname{emp}\left|x \mapsto x_{1}, \ldots, x_{n}\right| \mathrm{P}\left(x_{1}, \ldots, x_{n}\right) \mid \Sigma_{1} * \Sigma_{2} & & \text { Spatial assertions } \\
F::= & \Sigma|\Pi| \Sigma \wedge \Pi \mid \exists x . F & & \text { SL } \mid \Pi \text { assertions }
\end{array}
$$

Fig. 5: Syntax of assertions in $\mathrm{SL}_{\mathrm{ID}}$

Definition 2 (Syntactic equivalence). The syntactical equivalence relation of two spatial assertions $\Sigma_{1}$ and $\Sigma_{2}$, denoted as $\Sigma_{1} \cong \Sigma_{2}$, is recursively defined as follows:

$$
\begin{aligned}
& \text { - emp } \cong \mathrm{emp} \quad-u \mapsto v_{1}, \ldots, v_{n} \cong u \mapsto v_{1}, \ldots, v_{n} \quad-\mathrm{P}\left(u_{1}, \ldots, u_{n}\right) \cong \mathrm{P}\left(u_{1}, \ldots, u_{n}\right) \\
& \text { - If } \Sigma_{1} \cong \Sigma_{1}^{\prime} \text { and } \Sigma_{2} \cong \Sigma_{2}^{\prime} \text {, then } \Sigma_{1} * \Sigma_{2} \cong \Sigma_{1}^{\prime} * \Sigma_{2}^{\prime} \text { and } \Sigma_{1} * \Sigma_{2} \cong \Sigma_{2}^{\prime} * \Sigma_{1}^{\prime}
\end{aligned}
$$

Semantics. The semantics of $\mathrm{SL}_{\mathrm{ID}}$ assertions are given in Fig. 6. Given a set Var of variables, Sort of sorts, Val of values and Loc $\subset$ Val of memory addresses, a model of an assertion consists of:

- a stack model $s$, which is a function $s: \operatorname{Var} \rightarrow$ Val. We write $\llbracket \Pi \rrbracket_{s}$ to denote valuation of a pure assertion $\Pi$ under the stack model $s$. Note that the constant nil $\in \mathrm{Val} \backslash$ Loc denotes dangling memory address.

- a heap model $h$, which is a partial function $h$ : Loc $\rightarrow_{\text {fin }}$ (Sort $\rightarrow$ (Val list)). $\operatorname{dom}(h)$ denotes domain of $h$, and $|h|$ is cardinality of $\operatorname{dom}(h)$. We follow Reynolds' semantics [27] to consider finite heap models, i.e., $|h|<\infty . h \# h^{\prime}$ indicates that $h$ and $h^{\prime}$ have disjoint domains, i.e., $\operatorname{dom}(h) \cap \operatorname{dom}\left(h^{\prime}\right)=\varnothing$, and $h \circ h^{\prime}$ is the union of two disjoint heap models $h, h^{\prime}$, i.e., $h \# h^{\prime}$.

\subsection{Entailments in $\mathrm{SL}_{\mathrm{ID}}$}

In this section, we formally define the $\mathrm{SL}_{\mathrm{ID}}$ entailments and introduce a new concept of model of entailments, which will be used in the next section to construct the well-founded relation in our induction principle.

Definition 3 (Entailment). An entailment between two assertions $F$ and $G$, denoted as $F \vdash G$, is said to be valid (holds), iff $s, h \models F$ implies that $s, h \models G$, for all models $s, h$. Formally,

$$
F \vdash G \text { is valid, iff } \forall s, h .(s, h \models F \rightarrow s, h \models G)
$$

Here, $F$ and $G$ are respectively called the antecedent and the consequent of the entailment. For simplicity, the entailment $F \vdash G$ can be denoted by just $E$, i.e., $E \triangleq F \vdash G$. 


$$
\begin{array}{lcc}
s, h \models \Pi & \text { iff } & \llbracket \Pi \rrbracket_{s}=\text { true and } \operatorname{dom}(h)=\varnothing \\
s, h \models \mathrm{emp} & \text { iff } & \operatorname{dom}(h)=\varnothing \\
s, h \models x \mapsto x_{1}, \ldots, x_{n} & \text { iff } & s(x) \in \mathrm{Loc} \text { and } \operatorname{dom}(h)=\{s(x)\} \\
& & \text { and } h(s(x)) \iota=\left(s\left(x_{1}\right), \ldots, s\left(x_{n}\right)\right) \\
s, h \models \mathrm{P}\left(x_{1}, \ldots, x_{n}\right) & \text { iff } & s, h \models R_{i}\left(x_{1}, \ldots, x_{n}\right), \text { with } R_{i}\left(x_{1}, \ldots, x_{n}\right) \text { is one of } \\
& & \text { the definition cases of } \mathrm{P}\left(x_{1}, \ldots, x_{n}\right) \\
s, h \models \Sigma_{1} * \Sigma_{2} & \text { iff } & \text { there exist } h_{1}, h_{2} \text { such that: } h_{1} \# h_{2}, h_{1} \circ h_{2}=h \\
& & \text { and } s, h_{1} \models \Sigma_{1} \text { and } s, h_{2} \models \Sigma_{2} \\
s, h \models \Sigma \wedge \Pi & \text { iff } & \llbracket \Pi \rrbracket_{s}=\text { true and } s, h \models \Sigma \\
s, h \models \exists x . F & \text { iff } & \exists v \in \operatorname{Val} .[s \mid x: v], h \models F
\end{array}
$$

Fig. 6: Semantics of assertions in $\mathrm{SL}_{\mathrm{ID}} \cdot[f \mid x: y]$ is a function like $f$ except that it returns $y$ for input $x$.

Definition 4 (Model and counter-model). Given an entailment $E \triangleq F \vdash$ $G$. An SL model $s, h$ is called a model of $E$, iff $s, h \models F$ implies $s, h \models G$. On the contrary, $s, h$ is called a counter-model of $E$, iff $s, h \models F$ and $s, h \not \models G$.

We denote $s, h \models(F \vdash G)$, or $s, h \models E$, if $s, h$ is a model of $E$. Similarly, we write $s, h \forall(F \vdash G)$, or $s, h \forall E$, if $s, h$ is a counter-model of $E$. Given a list of $n$ entailments $E_{1}, \ldots, E_{n}$, we write $s, h \models E_{1}, \ldots, E_{n}$ if $s, h$ is a model of all $E_{1}, \ldots, E_{n}$, and $s, h \not \models E_{1}, \ldots, E_{n}$ if $s, h$ is a counter-model of some $E_{1}, \ldots, E_{n}$.

\section{Mutual induction proof for separation logic entailment using model order}

In this section, we first introduce the general schema of Noetherian induction, a.k.a. well-founded induction, and then apply it in proving SL entailments.

Noetherian induction [8]. Given a conjecture $\mathcal{P}(\alpha)$, with $\alpha$ is a structure of type $\tau$, the general schema of Noetherian induction on the structure $\alpha$ is

$$
\frac{\left.\forall \alpha: \tau .\left(\forall \beta: \tau . \beta \prec_{\tau} \alpha \rightarrow \mathcal{P}(\beta)\right) \rightarrow \mathcal{P}(\alpha)\right)}{\forall \alpha: \tau . \mathcal{P}(\alpha)}
$$

where $\prec_{\tau}$ is a well-founded relation on $\tau$, i.e., there is no infinite descending chain, like $\ldots \prec_{\tau} \alpha_{n} \prec_{\tau} \ldots \prec_{\tau} \alpha_{2} \prec_{\tau} \alpha_{1}$. Noetherian induction can be applied for arbitrary type $\tau$, such as data structures or control flow. However, success in proving a conjecture by induction is highly dependent on the choice of the induction variable $\alpha$ and the well-founded relation $\prec_{\tau}$.

Proving SL entailments using Noetherian induction. We observe that an SL entailment $E$ is said to be valid if $s, h \models E$ for all model $s, h$, given that the heap domain is finite, i.e., $\forall h .|h| \in \mathbb{N}$, according to Reynolds' semantics [27]. This inspires us to define a well-founded relation among SL models, called model 
order, by comparing size of their heap domains. To prove an SL entailment by Noetherian induction based on this order, we will show that if all the smaller models satisfying the entailment implies that the bigger model also satisfies the entailment, then the entailment is satisfied by all models, thus it is valid. The model order and induction principle are formally described as follows.

Definition 5 (Model order). The model order, denoted by $\prec$, of SL models is a binary relation defined as: $s_{1}, h_{1} \prec s_{2}, h_{2}$, if $\left|h_{1}\right|<\left|h_{2}\right|$.

Theorem 1 (Well-founded relation). The model order $\prec$ of SL models is a well-founded relation.

Proof. By contradiction, suppose that $\prec$ were not well-founded, then there would exist an infinite descending chain: $\ldots \prec s_{n}, h_{n} \prec \ldots \prec s_{1}, h_{1}$. It follows that there would exist an infinite descending chain: $\ldots<\left|h_{n}\right|<\ldots<\left|h_{1}\right|$. This is impossible since domain size of heap model is finite, i.e., $\left|h_{1}\right|, \ldots,\left|h_{n}\right|, \ldots \in \mathbb{N}$.

Theorem 2 (Induction principle). An entailment $E$ is valid, if for all model $s, h$, the following holds: $\left(\forall s^{\prime}, h^{\prime} . s^{\prime}, h^{\prime} \prec s, h \rightarrow s^{\prime}, h^{\prime} \models E\right) \rightarrow s, h \models E$. Formally:

$$
\frac{\forall s, h .\left(\forall s^{\prime}, h^{\prime} . s^{\prime}, h^{\prime} \prec s, h \rightarrow s^{\prime}, h^{\prime} \models E\right) \rightarrow s, h \models E}{\forall s, h . s, h \models E}
$$

Since our induction principle is constructed on the SL model order, an induction hypothesis can be used in the proof of any entailment whenever the decreasing condition on model order is satisfied. This flexibility allows us to extend the aforementioned principle to support mutual induction, in which multiple entailments can participate in an induction proof, and each of them can be used as a hypothesis to prove the other. In the following, we will introduce our mutual induction principle. Note that the induction principle in Theorem 2 is an instance of this principle, when only one entailment takes part in the induction proof.

Theorem 3 (Mutual induction principle). Given $n$ entailments $E_{1}, \ldots, E_{n}$. All of them are valid, if for all model $s, h$, the following holds: $\left(\forall s^{\prime}, h^{\prime} . s^{\prime}, h^{\prime} \prec\right.$ $\left.s, h \rightarrow s^{\prime}, h^{\prime} \models E_{1}, \ldots, E_{n}\right) \rightarrow s, h \models E_{1}, \ldots, E_{n}$. Formally:

$$
\frac{\forall s, h .\left(\forall s^{\prime}, h^{\prime} . s^{\prime}, h^{\prime} \prec s, h \rightarrow s^{\prime}, h^{\prime} \models E_{1}, \ldots, E_{n}\right) \rightarrow s, h \models E_{1}, \ldots, E_{n}}{\forall s, h . s, h \models E_{1}, \ldots, E_{n}}
$$

Proof. By contradiction, assume that some of $E_{1}, \ldots, E_{n}$ were invalid. Then, there would exist some counter-models $s, h$ such that $s, h \not E_{1}, \ldots, E_{n}$. Since $\prec$ is a well-founded relation, there would exist the least counter-model $s_{1}, h_{1}$ such that $s_{1}, h_{1} \not \models E_{1}, \ldots, E_{n}$, and, $s_{1}^{\prime}, h_{1}^{\prime} \models E_{1}, \ldots, E_{n}$ for all $s_{1}^{\prime}, h_{1}^{\prime} \prec s_{1}, h_{1}$. Following the theorem's hypothesis $\forall s, h .\left(\forall s^{\prime}, h^{\prime} . s^{\prime}, h^{\prime} \prec s, h \rightarrow s^{\prime}, h^{\prime} \models E_{1}, \ldots, E_{n}\right) \rightarrow s, h \models$ $E_{1}, \ldots, E_{n}$, we have $s_{1}, h_{1} \models E_{1}, \ldots, E_{n}$. This contradicts with the assumption that $s_{1}, h_{1}$ is a counter-model. 


\section{The proof system}

In this section, we introduce a sequent-based deductive system, which comprises a set of inference rules depicted in Fig. 7 (logical rules) and Fig. 8 (induction rules), and a proof search procedure in Fig. 10. Each inference rule has zero or more premises, a conclusion and possibly a side condition. A premise or a conclusion is described in the same form of $\mathcal{H}, \rho, F_{1} \vdash F_{2}$, where (i) $F_{1} \vdash F_{2}$ is an entailment, (ii) $\mathcal{H}$ is a set of entailments with validity status, which are recorded during proof search and can be used as hypotheses to prove $F_{1} \vdash F_{2}$, and (iii) $\rho$ is a proof trace capturing a chronological list of inference rules applied by the proof search procedure to reach $F_{1} \vdash F_{2}$.

In addition, the entailment in the conclusion of a rule is called the goal entailment. Rules with zero (empty) premise is called axiom rules. A proof trace $\rho$ containing $n$ rules $R_{1}, \ldots, R_{n}$, with $n \geq 0$, is represented by $\left[\left(R_{1}\right), \ldots,\left(R_{n}\right)\right]$, where the head $\left(R_{1}\right)$ of $\rho$ is the latest rule used by the proof search procedure. In addition, some operations over proof traces are (i) insertion: $(R):: \rho$, (ii) membership checking: $(R) \in \rho$, and (iii) concatenation: $\rho_{1} @ \rho_{2}$.

$$
\begin{aligned}
& \left(\perp \mathrm{L}_{1}\right) \overline{\mathcal{H}, \rho, F_{1} \wedge u \neq u \vdash F_{2}} \quad\left(\perp \mathrm{L}_{2}\right) \overline{\mathcal{H}, \rho, F_{1} * u \longmapsto \overrightarrow{\iota_{1}} \mapsto u \stackrel{\iota_{2}}{\longmapsto} \vdash F_{2}}
\end{aligned}
$$

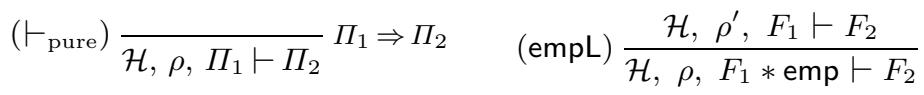

$$
\begin{aligned}
& (=\mathrm{L}) \frac{\mathcal{H}, \rho^{\prime}, F_{1}[u / v] \vdash F_{2}[u / v]}{\mathcal{H}, \rho, F_{1} \wedge u=v \vdash F_{2}} \quad(\text { empR }) \frac{\mathcal{H}, \rho^{\prime}, F_{1} \vdash \exists \vec{x} . F_{2}}{\mathcal{H}, \rho, F_{1} \vdash \exists \vec{x} .\left(F_{2} * \text { emp }\right)} \\
& (=\mathrm{R}) \frac{\mathcal{H}, \rho^{\prime}, F_{1} \vdash \exists \vec{x} . F_{2}}{\mathcal{H}, \rho, F_{1} \vdash \exists \vec{x} .\left(F_{2} \wedge u=u\right)}(* \mapsto) \frac{\mathcal{H}, \rho^{\prime}, F_{1} \vdash \exists \vec{x} .\left(F_{2} \wedge u=t \wedge \vec{v}=\vec{w}\right)}{\mathcal{H}, \rho, F_{1} * u \longmapsto^{\iota} \vec{v} \vdash \exists \vec{x} .\left(F_{2} * t \mapsto^{\iota} \vec{w}\right)}(u, \vec{v}) \# \vec{x} \\
& (\exists \mathrm{L}) \frac{\mathcal{H}, \rho^{\prime}, F_{1}[u / x] \vdash F_{2}}{\mathcal{H}, \rho, \exists x . F_{1} \vdash F_{2}} u \notin \mathrm{FV}\left(F_{2}\right) \quad(* \mathrm{P}) \frac{\mathcal{H}, \rho^{\prime}, F_{1} \vdash \exists \vec{x} .\left(F_{2} \wedge \vec{u}=\vec{v}\right)}{\mathcal{H}, \rho, F_{1} * \mathrm{P}(\vec{u}) \vdash \exists \vec{x} .\left(F_{2} * \mathrm{P}(\vec{v})\right)} \vec{u} \# \vec{x}
\end{aligned}
$$

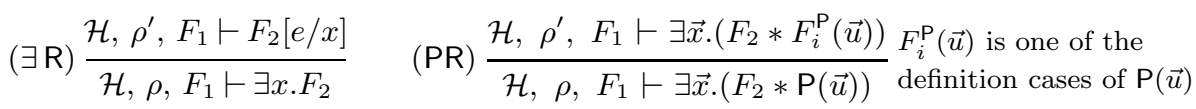

Fig. 7: Logical rules. Note that for a rule $R$ with trace $\rho$ in its conclusion, the trace in its premise is $\rho^{\prime} \triangleq(R):: \rho$.

\subsection{Logical rules}

Logical rules in Fig. 7 deal with the logical structure of SL entailments. For brevity, in these rules, we write the complete symbolic-heap assertion $\exists \vec{x}$. $(\Sigma \wedge \Pi)$ as a standalone $F$. We define the conjoined assertion $F * \Sigma^{\prime} \triangleq \Sigma * \Sigma^{\prime} \wedge \Pi$ and $F \wedge \Pi^{\prime} \triangleq \Sigma \wedge \Pi \wedge \Pi^{\prime}$, given that existential quantifiers does not occur in the outermost scope of $F$, i.e., $F \triangleq \Sigma \wedge \Pi$. The notation $\vec{u}=\vec{v}$ means 
$\left(u_{1}=v_{1}\right) \wedge \ldots \wedge\left(u_{n}=v_{n}\right)$, given that $\vec{u}=u_{1}, \ldots, u_{n}$ and $\vec{v}=v_{1}, \ldots, v_{n}$ are two lists containing the same number of variables. We also write $\vec{x} \# \vec{y}$ to denote $\vec{x}$ and $\vec{y}$ are disjoint, i.e., $\nexists u .(u \in \vec{x} \wedge u \in \vec{y})$, and use $\mathrm{FV}(F)$ to denote the list of all free variables of an assertion $F$. Moreover, $F[e / x]$ is a formula obtained from $F$ by substituting the expression $e$ for all occurrences of the free variable $x$ in $F$.

The set of logical rules are explained in details as follows:

- Axiom rules. The rule $\vdash_{\text {pure }}$ proves a pure entailment $\Pi_{1} \vdash \Pi_{2}$ by invoking off-the-shelf provers such as Z3 [18] to check the pure implication $\Pi_{1} \Rightarrow \Pi_{2}$ in its side condition. The two rules $\perp \mathrm{L}_{1}$ and $\perp \mathrm{L}_{2}$ decide an entailment vacuously valid if its antecedent is unsatisfiable, i.e., the antecedent contains a contradiction $(u \neq u)$ or overlaid data nodes $\left(u \stackrel{\iota_{1}}{\longmapsto} \vec{v} * u \stackrel{\iota_{2}}{\longmapsto} \vec{w}\right)$.

- Normalization rules. These rules simplify their goal entailments by either eliminating existentially quantified variables $(\exists L, \exists R)$, or removing equalities $(=\mathrm{L},=\mathrm{R})$ or empty heap predicates (empL, empR) from antecedents (left side) or consequents (right side) of the entailments.

- Frame rules. The two rules $* \mapsto$ and $* \mathrm{P}$ applies the frame property of SL [27] to remove identical spatial atoms from two sides of entailments. Note that the identical condition is guaranteed by adding equality constraints of these spatial atoms' arguments into consequents of the derived entailments.

- Unfolding rules. The rule PR derives a new entailment by unfolding a heap predicate in the goal entailment's consequent by its inductive definition. Note that unfolding a heap predicate in the entailment's antecedent will be performed by the induction rule Ind, as discussed in the next section.

\subsection{Induction rules}

$$
\begin{aligned}
& \text { (Ind) } \frac{\mathcal{H} \cup\{(H, ?)\}, \rho^{\prime}, F_{1} * F_{1}^{\mathrm{P}}(\vec{u}) \vdash F_{2} \quad \ldots \quad \mathcal{H} \cup\{(H, ?)\}, \rho^{\prime}, F_{1} * F_{m}^{\mathrm{P}}(\vec{u}) \vdash F_{2}}{\mathcal{H}, \rho, F_{1} * \mathrm{P}(\vec{u}) \vdash F_{2}} \dagger_{\text {(Ind) }} \\
& \text { Given } H \triangleq F_{1} * \mathrm{P}(\vec{u}) \vdash F_{2}, \rho^{\prime}=(\text { Ind }):: \rho \text {, and } \dagger_{(\text {Ind })}: \mathrm{P}(\vec{u}) \triangleq F_{1}^{\mathrm{P}}(\vec{u}) \vee \ldots \vee F_{m}^{\mathrm{P}}(\vec{u})
\end{aligned}
$$

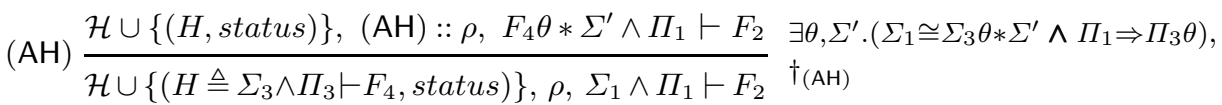

$$
\begin{aligned}
& \text { with } \dagger_{(\mathrm{AH})}:(\text { status }=\checkmark) \vee \exists \iota, u, \vec{v}, \Sigma^{\prime \prime} .\left(\Sigma^{\prime} \cong u \iota^{\iota} \vec{v} * \Sigma^{\prime \prime}\right) \\
& \vee \exists \rho_{1}, \rho_{2} .\left(\rho=\rho_{1} @[(* \mapsto)] @ \rho_{2} \wedge(\text { Ind }) \notin \rho_{1} \wedge(\text { Ind }) \in \rho_{2}\right) \text {. }
\end{aligned}
$$

Fig. 8: Induction rules

Fig. 8 presents inference rules implementing our mutual induction principle. The induction rule Ind firstly records its goal entailment as an induction hypothesis $H$, and unfolds an inductive heap predicate in the antecedent of $H$ to derive new entailments. When $H$ is inserted into the hypothesis vault $\mathcal{H}$, its status is initially assigned to ? (unknown), indicating that its validity is not known at the moment. Later, the status of $H$ will be updated to $\checkmark$ (valid) once the proof search procedure is able to prove it valid. Generally, given an entailment $E$ and 
its proof tree $\mathcal{T}$, the proof search procedure concludes that $E$ is valid if (i) every leaf of $\mathcal{T}$ is empty via applications of axiom rules, and (ii) all hypotheses used by the apply hypothesis rule $\mathrm{AH}$ must be derived in $\mathcal{T}$.

Rule $\mathrm{AH}$ is the key rule of our mutual induction principle, which applies an appropriate hypothesis $H \triangleq \Sigma_{3} \wedge \Pi_{3} \vdash F_{4}$ in proving its goal entailment $E \triangleq \Sigma_{1} \wedge \Pi_{1} \vdash F_{2}$. The rule firstly unifies the antecedents of $H$ and $E$ by a substitution $\theta$, i.e., there exists a spatial assertion $\Sigma^{\prime}$ such that $\Sigma_{1} \cong \Sigma_{3} \theta * \Sigma^{\prime}$ and $\Pi_{1} \Rightarrow \Pi_{3} \theta$. If such $\theta$ and $\Sigma^{\prime}$ exist, we can weaken the antecedent of $E$ as follows $\left(\Sigma_{1} \wedge \Pi_{1}\right) \vdash\left(\Sigma_{3} \theta * \Sigma^{\prime} \wedge \Pi_{3} \theta \wedge \Pi_{1}\right) \vdash\left(F_{4} \theta * \Sigma^{\prime} \wedge \Pi_{1}\right)$. Note that we use Reynolds's substitution law [27] to obtain $\Sigma_{3} \theta \wedge \Pi_{3} \theta \vdash F_{4} \theta$ from the hypothesis $H$. The proof system then derives the next goal entailment $F_{4} \theta * \Sigma^{\prime} \wedge \Pi_{1} \vdash F_{2}$ as shown in the premise of rule $\mathrm{AH}$.

The side condition $\dagger_{(\mathrm{AH})}$ of rule $\mathrm{AH}$ ensures the decreasing condition of the mutual induction principle. In particular, suppose that the proof search procedure applies a hypothesis $H$ in $\mathcal{H}$ to prove an entailment $E$ via rule $\mathrm{AH}$. If the status of $H$ is $\checkmark$, denoted by the first condition in $\dagger_{(\mathrm{AH})}$, then $H$ is already proved to be valid; thus it can

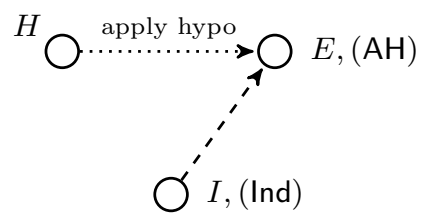

Fig. 9: Applying hypothesis be freely used to prove other entailments. Otherwise, the status of $H$ is ?, and $H$ may participate in a (mutual) induction proof with an entailment $I$ in the proof path of $E$, as depicted in Fig. 9. Note that the entailment $I$ has been recorded earlier as an induction hypothesis by an application of the induction rule Ind.

In the latter case, the induction principle requires the decrease of model size when applying the hypothesis $H$ to prove entailment $I$. We then show that this decreasing condition holds if one of the following conditions of $\dagger_{(\mathrm{AH})}$ is satisfied.

(i) $\exists \iota, u, \vec{v}, \Sigma^{\prime \prime} .\left(\Sigma^{\prime} \cong u \iota^{\iota} \vec{v} * \Sigma^{\prime \prime}\right)$ indicates that the left-over heap part $\Sigma^{\prime}$ after unifying antecedent of $H$ into that of $E$ contains at least one singleton heap predicate, or

(ii) $\exists \rho_{1}, \rho_{2} .\left(\rho=\rho_{1} @[(* \mapsto)] @ \rho_{2} \wedge\right.$ (Ind $\notin \notin \rho_{1} \wedge($ Ind $\left.) \in \rho_{2}\right)$ requires that there is a removal step of a singleton heap predicate by the rule $* \mapsto$ applied between this hypothesis application $\mathrm{AH}$ and the most recent induction step Ind.

Consider an arbitrary model $s, h$ satisfying $I$. During the derivation path from $I$ to $E$, the model $s, h$ is transformed into a corresponding model $s_{e}, h_{e}$ of $E$. We always have $\left|h_{e}\right| \leq|h|$ as the applications of logical rules and rule Ind never increase heap model size of entailments. Moreover, when applying $H$ to prove $E$, the model $s^{\prime}, h^{\prime}$ of $H$, which corresponds to $s_{e}, h_{e}$ of $E$, satisfies $\left|h^{\prime}\right| \leq\left|h_{e}\right|$, due to the unification step in rule $\mathrm{AH}$. We consider two following cases. If condition (i) is satisfied, then heap model size of the left-over part $\Sigma^{\prime}$ is at least 1 since $\Sigma^{\prime}$ contains a singleton heap predicate. As a result, $\left|h^{\prime}\right|<\left|h_{e}\right|$ and it follows that $\left|h^{\prime}\right|<|h|$. If condition (ii) is satisfied, then $\left|h_{e}\right|<|h|$ since there is a singleton heap predicate, whose size of heap model is 1 , is removed when deriving $I$ to 
$E$. This implies that $\left|h^{\prime}\right|<|h|$. In summary, we obtain that $\left|h^{\prime}\right|<|h|$ for both cases; thus, $s^{\prime}, h^{\prime} \prec s, h$. This concludes our explanation about the rule $\mathrm{AH}$.

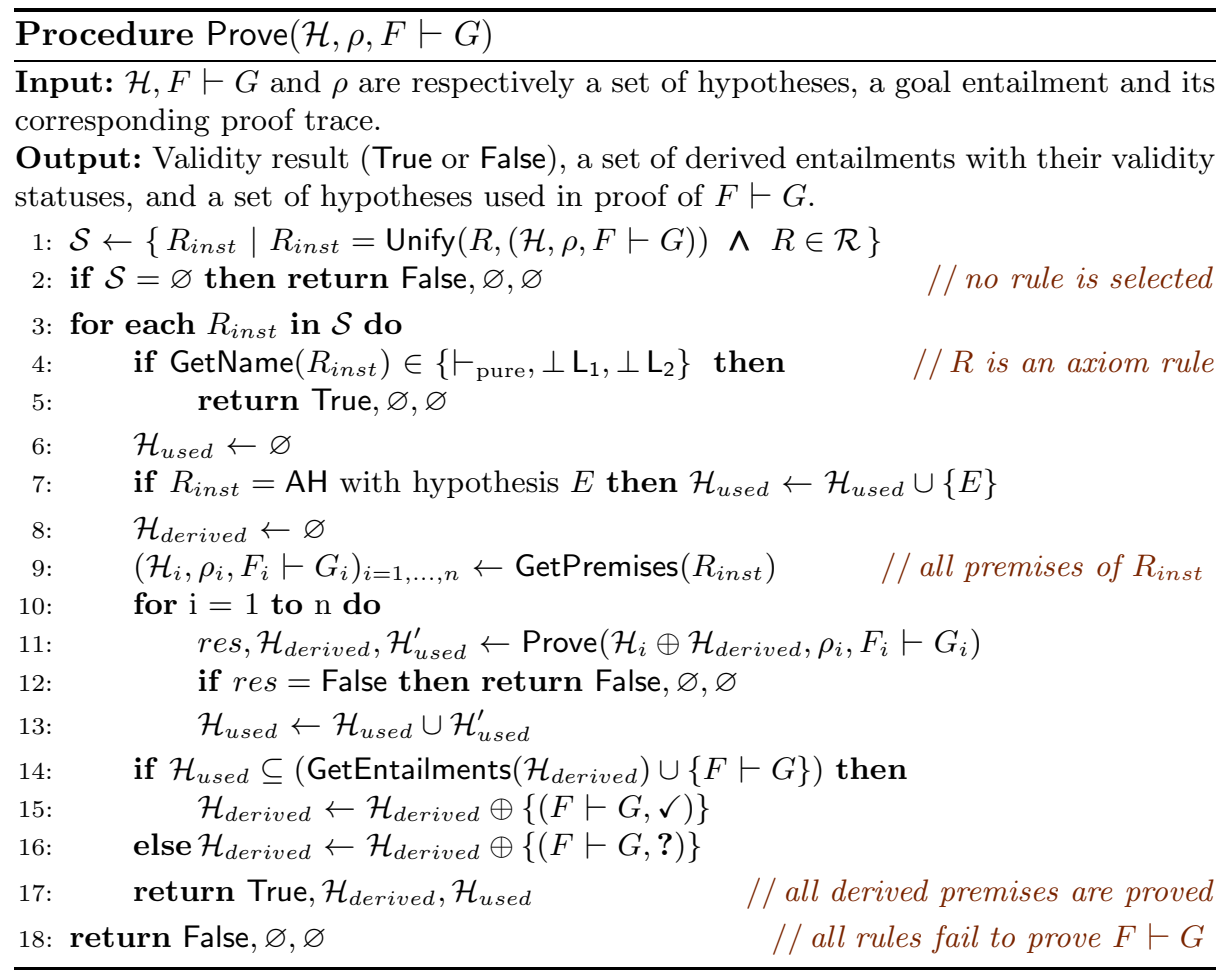

Fig. 10: General proof search procedure, in which $\mathcal{R}$ is the set of inference rules given in Fig. 7 and 8.

\subsection{Proof search procedure}

Our proof search procedure Prove is designed in a self-recursive manner, as presented in Fig. 10. Its inputs consist of a set of hypotheses, a proof trace, and an entailment, which are components of an inference rule's conclusion. To prove a candidate entailment $F \vdash G$, initially the hypothesis set $\mathcal{H}$ and the proof trace are assigned to empty ( $\varnothing$ and []).

Firstly, the procedure Prove finds a set $\mathcal{S}$ of suitable rules, whose conclusion can be unified with the goal entailment $F \vdash G$, among all inference rules in $\mathcal{R}$ (line 1). If no suitable rule is found, the procedure immediately returns False, indicating that it is unable to prove the entailment (line 2). Otherwise, it subsequently processes each discovered rule $R_{\text {inst }}$ in $\mathcal{S}$ by either (i) returning True to announce a valid result, if an axiom rule is selected (line 5), or (ii) recursively searching for proofs of the derived entailments in the premises of $R_{\text {inst }}$ (lines 9-17). In the latter case, the procedure returns False if one of the derived entailments is not proved (line 12), or returns True if all of them are proved (line 17). Finally, it 
simply returns False when it cannot prove the goal entailment with all selected rules (line 18).

The procedure uses a local variable $\mathcal{H}_{\text {used }}$ to store all hypotheses used during the proof search. $\mathcal{H}_{\text {used }}$ is updated when the rule $\mathrm{AH}$ is applied (line 7 ) or after the procedure finishes proving a derived entailment (lines 11 and 13). We also use another variable $\mathcal{H}_{\text {derived }}$ to capture all generated entailments with their validity statuses. The condition at line 14 checks if all hypotheses used to prove the entailment $F \vdash G$ are only introduced during the entailment's proof. If this condition is satisfied, then $F \vdash G$ is updated with a valid status $\checkmark$ (line 15). Otherwise, the entailment may participate in a (mutual) induction proof, thus its status is assigned to unknown? (line 16).

At line 11, the procedure uses not only the hypothesis set $\mathcal{H}_{i}$, introduced by the selected inference rule, but also the set $\mathcal{H}_{\text {derived }}$ containing entailments derived during proof search to prove a new goal entailment $F_{i} \vdash G_{i}$. This reflects our mutual induction principle which allows derived entailments to be used as hypotheses in other entailments' proofs. Note that the union and update operator $\oplus$ used in the algorithm will insert new entailments and their statuses into the set of hypotheses, or update the existing entailments with their new statuses. In addition, the auxiliary procedures used in our proof search procedure are named in a self-explanatory manner. In particular, Unify, GetName and GetPremises respectively unifies an inference rule with a goal entailment, or returns name and premises of an inference rule. Finally, GetEntailments returns all entailments stored in the set of derived entailments $\mathcal{H}_{\text {derived }}$.

Soundness. Soundness of our proof system is stated in Theorem 4. Due to page constraint, we present the detailed proof in the Appendix A

Theorem 4 (Soundness). Given an entailment E, if the proof search procedure returns True when proving $E$, then $E$ is valid.

\section{Experiment}

We have implemented the proposed induction proof technique into a prototype prover, named Songbird. The proof system and this paper's artifact are available for both online use and download at http://loris-5.d2.comp.nus.edu.sg/songbird/.

\begin{tabular}{|l|c|c|c|c|c|c|}
\hline \multicolumn{2}{|c|}{ Category } & Slide & Spen & Sleek & Cyclist & Songbird \\
\hline singly-II & $(64)$ & 12 & 3 & 48 & $\mathbf{6 3}$ & $\mathbf{6 3}$ \\
doubly-II & $(37)$ & 14 & 0 & 17 & 24 & $\mathbf{2 6}$ \\
nested-II & $(11)$ & 0 & $\mathbf{1 1}$ & 5 & 6 & $\mathbf{1 1}$ \\
skip-list & $(13)$ & 0 & $\mathbf{1 2}$ & 4 & 5 & 7 \\
tree & $(26)$ & 12 & 1 & 14 & 18 & $\mathbf{2 2}$ \\
\hline \hline Total & $(151)$ & 38 & 27 & 88 & 116 & $\mathbf{1 2 9}$ \\
\hline
\end{tabular}

(a)

\begin{tabular}{|l|c|c|c|c|}
\hline \multirow{2}{*}{} & \multicolumn{4}{|c|}{ Songbird } \\
\cline { 2 - 5 } & $\boldsymbol{V}_{\mathrm{sb}} \boldsymbol{X}_{\mathrm{o}}$ & $\boldsymbol{x}_{\mathrm{sb}} \boldsymbol{V}_{\mathrm{o}}$ & $\boldsymbol{V}_{\mathrm{sb}} \boldsymbol{V}_{\mathrm{o}}$ & $\boldsymbol{x}_{\mathrm{sb}} \boldsymbol{x}_{\mathrm{o}}$ \\
\hline Cyclist & 13 & 0 & 116 & 22 \\
\hline Sleek & 41 & 0 & 88 & 22 \\
\hline Spen & 109 & 7 & 20 & 15 \\
\hline Slide & 103 & 12 & 26 & 10 \\
\hline
\end{tabular}

(b)

Fig. 11: Overall evaluation on the benchmark slrd_entl of SL-COMP 
To evaluate our technique, we compared our system against state-of-the-art SL provers, including Slide [15,16], Spen [13], Sleek [10] and Cyclist [5,7], which had participated in the recent SL competition SL-COMP [30]. We are however unable to make direct comparison with the induction-based proof technique presented in [11] as their prover was not publicly available. Our evaluation was performed on an Ubuntu 14.04 machine with CPU Intel E5-2620 (2.4GHz) and RAM 64GB.

Firstly, we conduct the experiment on a set of valid entailments ${ }^{2}$, collected from the benchmark slrd_entl ${ }^{3}$ of SL-COMP. These entailments contain general inductive heap predicates denoting various data structures, such as singly linked lists (singly-II), doubly linked lists (doubly-II), nested lists (nested-II), skip lists (skiplist) and trees (tree). We then categorize problems in this benchmark based on their predicate types. In Fig. 11(a), we report the number of entailments successfully proved by a prover in each category, with a timeout of 30 seconds for proving an entailment. For each category, the total number of problems is put in parentheses, and the maximum number of entailments that can be proved by the list of provers are highlighted in bold. As can be seen, Songbird can prove more entailments than all the other tools. In particular, we are the best in almost categories, except for skip-list. However, in this category, we are behind only Spen, which has been specialized for skip lists [13]. Our technique might require more effective generalization to handle the unproven skip-list examples.

In Fig. 11(b), we make a detailed comparison among Songbird and other provers. Specifically, the first column $\left(\boldsymbol{V}_{\mathrm{sb}} \boldsymbol{X}_{\mathrm{o}}\right)$ shows the number of entailments that Songbird can prove valid whereas the others cannot. The second column $\left(\boldsymbol{x}_{\mathrm{sb}} \boldsymbol{V}_{\mathrm{o}}\right)$ reports the number of entailments that can be proved by other tools, but not by Songbird. The last two columns list the number of entailments that both Songbird and others can $\left(\boldsymbol{V}_{\mathrm{sb}} \boldsymbol{V}_{\mathrm{o}}\right)$ or cannot $\left(\boldsymbol{x}_{\mathrm{sb}} \boldsymbol{X}_{\mathrm{o}}\right)$ prove. We would like to highlight that our prover efficiently proves all entailments proved by Cyclist (resp. Sleek) in approximately half the time, i.e., 20.92 vs 46.40 seconds for 116 entailments, in comparison with Cyclist (resp. 8.38 vs 15.50 seconds for 88 entailments, in comparison with Sleek). In addition, there are 13 (resp. 41) entailments that can be proved by our tool, but not by Cyclist (resp. Sleek). Furthermore, our Songbird outperforms Spen and Slide by more than $65 \%$ of the total entailments, thanks to the proposed mutual induction proof technique.

Secondly, we would like to highlight the efficiency of mutual induction in our proof technique via a comparison between Songbird and its variant Songbird ${ }_{\mathrm{SI}}$, which exploits only induction hypotheses found within a single proof path. This mimics the structural induction technique which explores induction hypotheses in the same proof path. For this purpose, we designed a new entailment benchmark, namely slrd_ind, whose problems are more complex than those in the slrd_entl benchmark. For example, our handcrafted benchmark ${ }^{4}$ contains an

\footnotetext{
${ }^{2}$ We exclude the set of invalid entailments because some evaluated proof techniques, such as $[10,5]$, aim to only prove validity of entailments.

${ }^{3}$ Available at https://github.com/mihasighi/smtcomp14-sl/tree/master/bench.

4 The full benchmark is available at http://loris-5.d2.comp.nus.edu.sg/songbird/.
} 
entailment IsEven $(x, y) * y \mapsto z * \operatorname{IsEven}(z, t) \vdash \exists u$. IsEven $(x, u) * u \mapsto t$ with the predicate IsEven $(x, y)$ denoting list segments with even length. This entailment was inspired by the entailment IsEven $(x, y) * \operatorname{Is} \operatorname{Even}(y, z) \vdash \operatorname{IsEven}(x, z)$ in the problem 11.tst.smt2 of sIrd_entl, contributed by team Cyclist. Note that entailments in our benchmark were constructed on the same set of linked list predicates provided in slrd_entl, comprised of regular singly linked lists (II), linked lists with even or odd length (II-even/odd) and linked list segments which are left- or right-recursively defined (II-left/right). We also use a new II2 list segment predicate whose structure is similar to the predicate tmp in our motivating example. In addition, problems in the misc. category involve all aforementioned linked list predicates.

As shown in Fig. 12, Songbird ${ }_{\text {SI }}$ is able to prove nearly $70 \%$ of the total entailments, which is slightly better than Cyclist ${ }^{5}$, whereas Songbird, with full capability of mutual induction, can prove the whole set of entailments. This result is encouraging as it shows the usefulness and essentials of our mutual explicit induction proof technique in proving SL entailments.

\begin{tabular}{|c|c|c|c|}
\hline Category & Cyclist & Songbird $_{\mathrm{SI}}$ & Songbird \\
\hline$\|/\| 2$ & 18 & 22 & 24 \\
\hline Il-even/odd (20) & 8 & 17 & 20 \\
\hline II-left/right (20) & 12 & 10 & 20 \\
\hline misc. & 17 & 16 & 32 \\
\hline Total & 55 & 65 & 96 \\
\hline
\end{tabular}

Fig. 12: Comparison on slrd_ind benchmark

\section{Conclusion}

We have proposed a novel induction technique and developed a proof system for automatically proving entailments in a fragment of SL with general inductive predicates. In essence, we show that induction can be performed on the size of the heap models of SL entailments. The implication is that, during automatic proof construction, the goal entailment and entailments derived in the entire proof tree can be used as hypotheses to prove other derived entailments, and vice versa. This novel proposal has opened up the feasibility of mutual induction in automatic proof, leading to shorter proof trees being built. In future, we would like to develop a verification system on top of the prover Songbird, so that our mutual explicit induction technique can be effectively used for automated verification of memory safety in imperative programs.

Acknowledgement. We would like to thank the anonymous reviewers for their valuable and helpful feedback. The first author would like to thank Dr. James Brotherston for the useful discussion about the cyclic proof. This work has been supported by NUS Research Grant R-252-000-553-112. Ton Chanh and WeiNgan are partially supported by MoE Tier-2 grant MOE2013-T2-2-146.

\footnotetext{
${ }^{5}$ We do not list other provers in Fig. 12 as they cannot prove any problems in slrd_ind.
} 


\section{References}

1. Infer: A tool to detect bugs in Android and iOS apps before they ship. http://fbinfer.com/, accessed: 2016-05-27

2. Berdine, J., Calcagno, C., O'Hearn, P.W.: A Decidable Fragment of Separation Logic. In: International Conference on Foundations of Software Technology and Theoretical Computer Science (FSTTCS). pp. 97-109 (2004)

3. Berdine, J., Calcagno, C., O'Hearn, P.W.: Symbolic Execution with Separation Logic. In: Asian Symposium on Programming Languages and Systems (APLAS). pp. 52-68 (2005)

4. Bozga, M., Iosif, R., Perarnau, S.: Quantitative Separation Logic and Programs with Lists. J. Autom. Reasoning 45(2), 131-156 (2010)

5. Brotherston, J., Distefano, D., Petersen, R.L.: Automated Cyclic Entailment Proofs in Separation Logic. In: International Conference on Automated Deduction (CADE). pp. 131-146 (2011)

6. Brotherston, J., Gorogiannis, N., Kanovich, M.I., Rowe, R.: Model checking for Symbolic-Heap Separation Logic with inductive predicates. In: Symposium on Principles of Programming Languages (POPL). pp. 84-96 (2016)

7. Brotherston, J., Gorogiannis, N., Petersen, R.L.: A generic cyclic theorem prover. In: Asian Symposium on Programming Languages and Systems (APLAS). pp. 350-367 (2012)

8. Bundy, A.: The automation of proof by mathematical induction. In: Handbook of Automated Reasoning (in 2 volumes). pp. 845-911 (2001)

9. Calcagno, C., Distefano, D., O'Hearn, P.W., Yang, H.: Compositional shape analysis by means of bi-abduction. In: Symposium on Principles of Programming Languages (POPL). pp. 289-300 (2009)

10. Chin, W., David, C., Nguyen, H.H., Qin, S.: Automated verification of shape, size and bag properties via user-defined predicates in Separation Logic. Science of Computer Programming (SCP) 77(9), 1006-1036 (2012)

11. Chu, D., Jaffar, J., Trinh, M.: Automatic induction proofs of data-structures in imperative programs. In: Conference on Programming Language Design and Implementation (PLDI). pp. 457-466 (2015)

12. Cook, B., Haase, C., Ouaknine, J., Parkinson, M.J., Worrell, J.: Tractable Reasoning in a Fragment of Separation Logic. In: International Conference on Concurrency Theory (CONCUR). pp. 235-249 (2011)

13. Enea, C., Lengál, O., Sighireanu, M., Vojnar, T.: Compositional Entailment Checking for a Fragment of Separation Logic. In: Asian Symposium on Programming Languages and Systems (APLAS). pp. 314-333 (2014)

14. Enea, C., Sighireanu, M., Wu, Z.: On automated lemma generation for separation logic with inductive definitions. In: International Symposium on Automated Technology for Verification and Analysis (ATVA). pp. 80-96 (2015)

15. Iosif, R., Rogalewicz, A., Simácek, J.: The Tree Width of Separation Logic with Recursive Definitions. In: International Conference on Automated Deduction (CADE). pp. 21-38 (2013)

16. Iosif, R., Rogalewicz, A., Vojnar, T.: Deciding Entailments in Inductive Separation Logic with Tree Automata. In: International Symposium on Automated Technology for Verification and Analysis (ATVA). pp. 201-218 (2014)

17. Le, Q.L., Gherghina, C., Qin, S., Chin, W.: Shape analysis via second-order biabduction. In: International Conference on Computer Aided Verification (CAV). pp. 52-68 (2014) 
18. Moura, L.M.D., Bjørner, N.: Z3: An Efficient SMT Solver. In: International Conference on Tools and Algorithms for Construction and Analysis of Systems (TACAS). pp. 337-340 (2008)

19. Nguyen, H.H., Chin, W.: Enhancing Program Verification with Lemmas. In: International Conference on Computer Aided Verification (CAV). pp. 355-369 (2008)

20. Nguyen, H.H., David, C., Qin, S., Chin, W.: Automated verification of shape and size properties via Separation Logic. In: Int. Conf. on Verification, Model Checking, and Abstract Interpretation (VMCAI). pp. 251-266 (2007)

21. O'Hearn, P.W., Reynolds, J.C., Yang, H.: Local Reasoning about Programs that Alter Data Structures. In: International Conference on Computer Science Logic (CSL). pp. 1-19 (2001)

22. Pérez, J.A.N., Rybalchenko, A.: Separation Logic + Superposition Calculus = Heap Theorem Prover. In: Conference on Programming Language Design and Implementation (PLDI). pp. 556-566 (2011)

23. Pérez, J.A.N., Rybalchenko, A.: Separation Logic Modulo Theories. In: Asian Symposium on Programming Languages and Systems (APLAS). pp. 90-106 (2013)

24. Piskac, R., Wies, T., Zufferey, D.: Automating Separation Logic Using SMT. In: International Conference on Computer Aided Verification (CAV). pp. 773-789 (2013)

25. Piskac, R., Wies, T., Zufferey, D.: Automating Separation Logic with Trees and Data. In: International Conference on Computer Aided Verification (CAV). pp. 711-728 (2014)

26. Qiu, X., Garg, P., Stefanescu, A., Madhusudan, P.: Natural proofs for structure, data, and separation. In: Conference on Programming Language Design and Implementation (PLDI). pp. 231-242 (2013)

27. Reynolds, J.C.: An Introduction to Separation Logic - Lecture Notes for the PhD Fall School on Logics and Semantics of State, Copenhagen 2008. URL:http://www.cs.cmu.edu/ jcr/copenhagen08.pdf, [Online; accessed 20-Jan2016]

28. Reynolds, J.C.: Intuitionistic Reasoning about Shared Mutable Data Structure. In: Millennial Perspectives in Computer Science. pp. 303-321. Palgrave (2000)

29. Reynolds, J.C.: Separation Logic: A Logic for Shared Mutable Data Structures. In: Symposium on Logic in Computer Science (LICS). pp. 55-74 (2002)

30. Sighireanu, M., Cok, D.R.: Report on SL-COMP 2014. Journal of Satisfiability, Boolean Modeling and Computation (2014)

31. Ta, Q.T., Le, T.C., Khoo, S.C., Chin, W.N.: Automated Mutual Explicit Induction Proof in Separation Logic. arXiv abs/1609.00919 (2016) 


\section{A Soundness proof}

\section{A.1 Soundness of inference rules}

We prove soundness of these rules by showing that if entailments in their premises are valid, and their side conditions are satisfied, then goal entailments in their conclusions are also valid.

1. Axiom rules $\perp L_{1}, \perp L_{2}$ and $\vdash_{\text {pure }}$ :

$$
\begin{aligned}
& \left(\perp \mathrm{L}_{1}\right) \overline{\mathcal{H}, \rho, F_{1} \wedge u \neq u \vdash F_{2}} \quad\left(\perp \mathrm{L}_{2}\right) \overline{\mathcal{H}, \rho, F_{1} * u \stackrel{\iota_{1}}{\longmapsto} \vec{v} * u \stackrel{\iota^{2}}{\longmapsto} \vec{w} \vdash F_{2}} \\
& \left(\vdash_{\text {pure }}\right) \frac{\overline{\mathcal{H}, \rho, \Pi_{1} \vdash \Pi_{2}}}{\Pi_{1} \Rightarrow \Pi_{2}}
\end{aligned}
$$

- It is easy to verify that antecedents of goal entailments in the two rules $\perp \mathrm{L}_{1}, \perp \mathrm{L}_{2}$ are unsatisfiable, since they either contain a contradiction $(u \neq u$ in the rule $\perp L_{1}$ ) or contain two data nodes having the same memory address $\left(u \stackrel{\iota_{1}}{\longmapsto} \vec{v} * u \stackrel{\iota_{2}}{\longmapsto} \vec{w}\right.$ in the rule $\left.\perp \mathrm{L}_{2}\right)$. Therefore, these entailments are evidently valid.

- When the rule $\vdash_{\text {pure }}$ is applied, pure provers will be invoked to check the side condition: $\Pi_{1} \Rightarrow \Pi_{2}$. If this side condition holds, then clearly the entailment $\Pi_{1} \vdash \Pi_{2}$ is valid.

2. Rule $=\mathrm{L}$ and $=\mathrm{R}$ :

$$
(=\mathrm{L}) \frac{\mathcal{H}, \rho^{\prime}, F_{1}[u / v] \vdash F_{2}[u / v]}{\mathcal{H}, \rho, F_{1} \wedge u=v \vdash F_{2}} \quad(=\mathrm{R}) \frac{\mathcal{H}, \rho^{\prime}, F_{1} \vdash \exists \vec{x} . F_{2}}{\mathcal{H}, \rho, F_{1} \vdash \exists \vec{x} .\left(F_{2} \wedge u=u\right)}
$$

- Soundness of the rule $=\mathrm{R}$ is evident since the condition $u=u$ in the goal entailment $F_{1} \vdash \exists \vec{x} .\left(F_{2} \wedge u=u\right)$ is a tautology.

- For the rule $=\mathrm{L}$, consider an arbitrary model $s, h$ of antecedent of the goal entailment. Then $s, h \models F_{1} \wedge u=v$. It follows that $s(u)=s(v)$, therefore $s, h \models$ $F_{1}[u / v]$. Since the entailment $F_{1}[u / v] \vdash F_{2}[u / v]$ in the rule's premise is valid, it implies $s, h \models F_{2}[u / v]$. But this also means that $s, h \models F_{2}$, since $s(u)=s(v)$. Therefore, $F_{1} \wedge u=v \vdash F_{2}$ is valid.

\section{Rule $\exists \mathrm{L}$ and $\exists \mathrm{R}$ :}

$$
(\exists \mathrm{L}) \frac{\mathcal{H}, \rho^{\prime}, F_{1}[u / x] \vdash F_{2}}{\mathcal{H}, \rho, \exists x . F_{1} \vdash F_{2}} u \notin \mathrm{Fv}\left(F_{2}\right) \quad(\exists \mathrm{R}) \frac{\mathcal{H}, \rho^{\prime}, F_{1} \vdash F_{2}[e / x]}{\mathcal{H}, \rho, F_{1} \vdash \exists x . F_{2}}
$$

- To prove correctness of the rule $\exists \mathrm{L}$, we consider an arbitrary model $s, h$ such that $s, h \models \exists x . F_{1}$. By semantics of the $\exists$ quantification, there is an integer value $v \in$ Int such that $s^{\prime}, h \models F_{1}$, with $s^{\prime}=[s \mid x: v]$. Then $s^{\prime \prime}, h \models F_{1}[u / x]$ with $s^{\prime \prime}$ is extended from $s^{\prime}$ such that $s^{\prime \prime}(u)=s^{\prime}(x)$. Since $s^{\prime}=[s \mid x: v]$, it follows that $s^{\prime \prime}=[s \mid u: v]$. On the other hand, given that entailment in the rule's premise is valid, then $s^{\prime \prime}, h \models F_{2}$. It implies that $[s \mid u: v] \models F_{2}$. In addition $u \notin \mathrm{FV}\left(F_{2}\right)$. 
Therefore $s, h \models F_{2}$. Since $s, h$ is chosen arbitrarily, it follows that the rule $\exists \mathrm{L}$ is correct.

- Correctness of $\exists \mathrm{R}$ is straight forward. Suppose that $s, h$ is an arbitrary model such that $s, h \models F_{1}$. Since entailment in the rule's premise is valid, then $s, h \models$ $F_{2}[e / x]$. It follows that $s, h$ also satisfies $\exists x . F_{2}$, by simply choosing value $v$ of $x$ such that $v=\llbracket e \rrbracket_{s}$. Since $s, h$ is chosen arbitrarily, it follows that the entailment in the rule's conclusion is valid. Therefore $\exists R$ is valid.

\section{Rule empL and empR:}

$$
(\mathrm{empL}) \frac{\mathcal{H}, \rho^{\prime}, F_{1} \vdash F_{2}}{\mathcal{H}, \rho, F_{1} * \text { emp } \vdash F_{2}} \quad(\mathrm{empR}) \frac{\mathcal{H}, \rho^{\prime}, F_{1} \vdash \exists \vec{x} . F_{2}}{\mathcal{H}, \rho, F_{1} \vdash \exists \vec{x} .\left(F_{2} * \text { emp }\right)}
$$

It is evident that two assertions $F_{1} *$ emp and $F_{1}$ in the rule empL are semantically equivalent. In addition, $F_{2} *$ emp and $F_{2}$ in the rule empR are also semantically equivalent. It follows that both the two rules empL and empR are correct.

\section{Rule $* \mapsto$ and $* \mathrm{P}$ :}

$$
\begin{gathered}
(* \mapsto) \frac{\mathcal{H}, \rho^{\prime}, F_{1} \vdash \exists \vec{x} .\left(F_{2} \wedge u=t \wedge \vec{v}=\vec{w}\right)}{\mathcal{H}, \rho, F_{1} * u \iota^{\iota} \vec{v} \vdash \exists \vec{x} .\left(F_{2} * t !^{\iota} \vec{w}\right)}(u, \vec{v}) \# \vec{x} \\
(* \mathrm{P}) \frac{\mathcal{H}, \rho^{\prime}, F_{1} \vdash \exists \vec{x} .\left(F_{2} \wedge \vec{u}=\vec{v}\right)}{\mathcal{H}, \rho, F_{1} * \mathrm{P}(\vec{u}) \vdash \exists \vec{x} .\left(F_{2} * \mathrm{P}(\vec{v})\right)} \vec{u} \# \vec{x}
\end{gathered}
$$

In the following, we present soundness proof of the rule $* \mapsto$. Soundness of $* \mathrm{P}$ can proved in a similar way.

Consider an arbitrary model $s, h$ such that $s, h \models F_{1} * u \longmapsto \vec{v}$. Then, there exists $h_{1} \# h_{2}$ such that $h=h_{1} \circ h_{2}$, and $s, h_{1} \models F_{1}$, and $s, h_{2} \vDash u \longmapsto \vec{v}$. On one hand, entailment in the rule's premise is valid, it follows that $s, h_{1} \models \exists \vec{x}$. $\left(F_{2} \wedge\right.$ $u=t \wedge \vec{v}=\vec{w})$. By semantics of $\exists$ quantification, $s^{\prime}, h_{1} \models F_{2} \wedge u=t \wedge \vec{v}=\vec{w}$, with $s^{\prime}$ is a model extended from $s$ with integer values of $\vec{x}$. On the other hand, the rule's side condition gives $u \notin \vec{x}$, and $\vec{v} \# \vec{x}$, and $s^{\prime}$ is extend from $s$ with values of $\vec{x}$, and $s, h_{2} \models u \longmapsto \vec{\iota}$, it follows that $s^{\prime}, h_{2} \models u \vdash \vec{v}$. Combining these two hands, with the fact that $h_{1} \# h_{2}$ and $h=h_{1} \circ h_{2}$, the following holds: $s^{\prime}, h \models F_{2} * u \stackrel{\iota}{\longmapsto} \vec{v} \wedge u=t \wedge \vec{v}=\vec{w}$. By semantics of equality (=), the following also holds: $s^{\prime}, h \models F_{2} * t \iota^{\iota} \vec{w} \wedge u=t \wedge \vec{v}=\vec{w}$. By weakening this assertion via dropping the condition $u=t \wedge \vec{v}=\vec{w}$, it is evident that $s^{\prime}, h \models F_{2} * t \stackrel{\iota}{\longmapsto} \vec{w}$. Since $s^{\prime}$ is extended from $s$ with values of $\vec{x}$, it is evident that $s, h \models \exists \vec{x} .\left(F_{2} * t \iota^{\iota} \mapsto \vec{w}\right)$. Recall that $s, h$ is chosen arbitrarily, this implies that the rule $* \mapsto$ is sound.

\section{Rule PR:}

(PR) $\frac{\mathcal{H}, \rho^{\prime}, F_{1} \vdash \exists \vec{x} .\left(F_{2} * F_{i}^{\mathrm{P}}(\vec{u})\right)}{\mathcal{H}, \rho, F_{1} \vdash \exists \vec{x} .\left(F_{2} * \mathrm{P}(\vec{u})\right)} \begin{aligned} & F_{i}^{\mathrm{P}}(\vec{u}) \text { is one of the } \\ & \text { definition cases of } \mathrm{P}(\vec{u})\end{aligned}$ 
Consider an arbitrary model $s, h$ such that $s, h \models F_{1}$. Since entailment in the rule's premise is valid, it follows that $s, h \models \exists \vec{x} .\left(F_{2} * F_{i}^{\mathrm{P}}(\vec{u})\right)$. In addition, the rule's side condition that $F_{i}^{\mathrm{P}}(\vec{u})$ is one of the definition cases of $\mathrm{P}(\vec{u})$ clearly implies that $s, h \models \exists \vec{x} .\left(F_{2} * \mathrm{P}(\vec{u})\right)$. Since $s, h$ is chosen arbitrarily, it follows that entailment in the rule's conclusion is valid.

\section{Rule Ind:}

$$
\text { (Ind) } \frac{\mathcal{H} \cup\{(H, ?)\}, \rho^{\prime}, F_{1} * F_{1}^{\mathrm{P}}(\vec{u}) \vdash F_{2} \quad \ldots \quad \mathcal{H} \cup\{(H, ?)\}, \rho^{\prime}, F_{1} * F_{m}^{\mathrm{P}}(\vec{u}) \vdash F_{2}}{\mathcal{H}, \rho, F_{1} * \mathrm{P}(\vec{u}) \vdash F_{2}} \dagger_{\text {(Ind) }}
$$

Given $H \triangleq F_{1} * \mathrm{P}(\vec{u}) \vdash F_{2}, \quad \rho^{\prime}=($ Ind $):: \rho$, and $\dagger_{(\text {Ind })}: \mathrm{P}(\vec{u}) \triangleq F_{1}^{\mathrm{P}}(\vec{u}) \vee \ldots \vee F_{m}^{\mathrm{P}}(\vec{u})$

We show that if all of the entailments $F_{1} * F_{1}^{\mathrm{P}}(\vec{u}) \vdash F_{2}, \ldots, F_{1} * F_{m}^{\mathrm{P}}(\vec{u}) \vdash F_{2}$ in the rule premise are valid, then so is the entailment $F_{1} * \mathrm{P}(\vec{u}) \vdash F_{2}$ in the conclusion.

Indeed, consider an arbitrary model $s, h$ such that $s, h \models F_{1} * \mathrm{P}(\vec{u})$. Side condition of the rule gives that $\mathrm{P}(\vec{u}) \triangleq F_{1}^{\mathrm{P}}(\vec{u}) \vee \ldots \vee F_{m}^{\mathrm{P}}(\vec{u})$, i.e., $F_{1}^{\mathrm{P}}(\vec{u}), \ldots, F_{m}^{\mathrm{P}}(\vec{u})$ are all definition cases of $\mathrm{P}(\vec{u})$. Since $s, h \models F_{1} * \mathrm{P}(\vec{u})$, it follows that $s, h \models F_{1} * F_{i}^{\mathrm{P}}(\vec{u})$, for all $i=1 \ldots m$. On the other hand, $F_{1} * F_{1}^{\mathrm{P}}(\vec{u}), \ldots, F_{1} * F_{m}^{\mathrm{P}}(\vec{u})$ are antecedents of all entailments in this rule's premises, and these entailments have the same consequent $F_{2}$. Therefore, $s, h \models F_{2}$. Since $s, h$ is chosen arbitrarily, it follows that entailment in the rule's conclusion is valid. This confirms soundness of the rule Ind.

\section{Rule AH:}

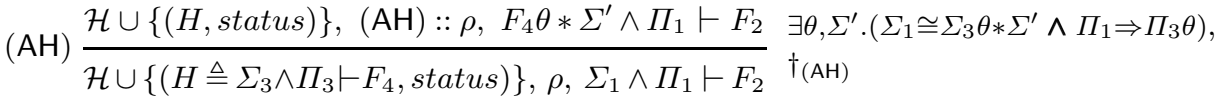

$$
\begin{aligned}
& \text { with } \dagger_{\text {(AH) }}:(\text { status }=\checkmark) \vee \exists \iota, u, \vec{v}, \Sigma^{\prime \prime} .\left(\Sigma^{\prime} \cong u \mapsto{ }^{\iota} \vec{v} * \Sigma^{\prime \prime}\right) \\
& \vee \exists \rho_{1}, \rho_{2} .\left(\rho=\rho_{1} @[(* \mapsto)] @ \rho_{2} \wedge \text { (Ind) } \notin \rho_{1} \wedge \text { (Ind) } \in \rho_{2}\right) .
\end{aligned}
$$

We show that if two entailments $F_{4} \theta * \Sigma^{\prime} \wedge \Pi_{1} \vdash F_{2}$ and $\Sigma_{3} \wedge \Pi_{3} \vdash F_{4}$ in the rule's premise are valid, then so is the entailment in the rule's conclusion.

Indeed, the side condition $\Sigma_{1} \cong \Sigma_{3} \theta * \Sigma^{\prime}$ and $\Pi_{1} \Rightarrow \Pi_{3} \theta$ implies that $\Sigma_{1} \wedge \Pi_{1} \vdash$ $\Sigma_{3} \theta * \Sigma^{\prime} \wedge \Pi_{3} \theta \wedge \Pi_{1}$ is valid.

By applying Reynolds's substitution law [27], the hypothesis $\Sigma_{3} \wedge \Pi_{3} \vdash F_{4}$ implies that $\Sigma_{3} \theta \wedge \Pi_{3} \theta \vdash F_{4} \theta$ is valid. It follows that the following entailment is also valid: $\Sigma_{3} \theta * \Sigma^{\prime} \wedge \Pi_{3} \theta \wedge \Pi_{1} \vdash F_{4} \theta * \Sigma^{\prime} \wedge \Pi_{1}$.

We have shown that the two entailments $\Sigma_{1} \wedge \Pi_{1} \vdash \Sigma_{3} \theta * \Sigma^{\prime} \wedge \Pi_{3} \theta \wedge \Pi_{1}$ and $\Sigma_{3} \theta * \Sigma^{\prime} \wedge \Pi_{3} \theta \wedge \Pi_{1} \vdash F_{4} \theta * \Sigma^{\prime} \wedge \Pi_{1}$ are valid. In addition, the rule's premise gives that $F_{4} \theta * \Sigma^{\prime} \wedge \Pi_{1} \vdash F_{2}$ is valid. It follows that the entailment $\Sigma_{1} \wedge \Pi_{1} \vdash F_{2}$ in the rule's conclusion is valid as well. Therefore, the rule AH is correct. 


\section{A.2 Soundness of the proof system}

We are now ready to prove soundness of our proof system, which is stated in Theorem 4: Given an entailment $E$, if the proof search procedure returns True when proving $E$, then $E$ is valid.

Our soundness proof is as follow.

Suppose that during proving the entailment $E$, the proof search procedure derives a proof tree $\mathcal{T}$ of $E$. If the rule $\mathrm{AH}$ (apply hypothesis) is not used in $\mathcal{T}$, then it is clear that induction is not used in proof of $E$. Then, it is clear that $E$ is valid, since soundness of our inference rules are proven in the previous section.

If the rule $\mathrm{AH}$ is used in $\mathcal{T}$, then statuses of the applied hypotheses, at the moment they are used, can be either valid $(\checkmark)$ or unknown (?). If statuses of all the applied hypotheses in the proof tree $\mathcal{T}$ are valid $(\checkmark)$, then it is clear that $E$ is valid, due to correctness of our inference rules. If there exists some applied hypotheses whose statuses are unknown (?), then these hypotheses may participate in a mutual induction proof. We will show that the entailment $E$ is also valid in this case.

Recall that hypotheses applied by the rule $\mathrm{AH}$ are either (i) induction hypotheses recorded by the rule Ind, or (ii) hypotheses derived by other rules during proof search. Therefore, in the proof tree $\mathcal{T}$, both the induction hypotheses and other hypotheses can participate in a mutual induction proof. We will transform the proof tree $\mathcal{T}$ into a new tree $\mathcal{T}^{\prime}$ in which the mutual induction proof involves only the induction hypotheses recorded by the rule Ind. This can be done by modifying the rule $\mathrm{AH}$ to put the used hypothesis, which is not derived by the rule Ind, into premises of the rule.

In particular, suppose the (simplified) rule $\mathrm{AH}$ which applies a hypothesis $\Sigma_{3} \wedge$ $\Pi_{3} \vdash F_{4}$, (not derived by the rule Ind), into proving a goal entailment $\Sigma_{1} \wedge \Pi_{1} \vdash$ $F_{2}$ as follow:

(AH) $\frac{F_{4} \theta * \Sigma^{\prime} \vdash F_{2}}{\Sigma_{1} \wedge \Pi_{1} \vdash F_{2}} \quad \begin{aligned} & \text { apply hypothesis: } \Sigma_{3} \wedge \Pi_{3} \vdash F_{4} \text {, (which is not derived by rule Ind) } \\ & \exists \theta, \Sigma^{\prime} .\left(\Sigma_{1} \equiv \Sigma_{3} \theta * \Sigma^{\prime} \wedge \Pi_{1} \Rightarrow \Pi_{3} \theta\right)\end{aligned}$

We modify the rule $\mathrm{AH}$ so that the hypothesis $\Sigma_{3} \wedge \Pi_{3} \vdash F_{4}$ appears in the premises as follows:

(modified AH) $\frac{\Sigma_{3} \wedge \Pi_{3} \vdash F_{4} \quad F_{4} \theta * \Sigma^{\prime} \vdash F_{2}}{\Sigma_{1} \wedge \Pi_{1} \vdash F_{2}} \begin{aligned} & \Sigma_{3} \wedge \Pi_{3} \vdash F_{4} \text { is not derived by rule Ind } \\ & \exists \theta, \Sigma^{\prime} .\left(\Sigma_{1} \equiv \Sigma_{3} \theta * \Sigma^{\prime} \wedge \Pi_{1} \Rightarrow \Pi_{3} \theta\right)\end{aligned}$ 


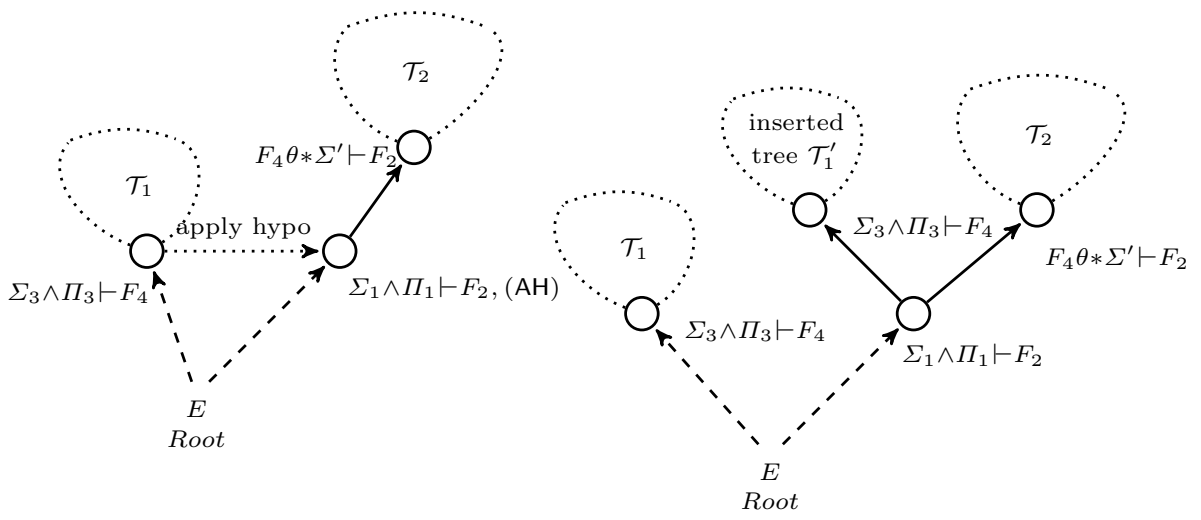

Fig. 13: Original proof tree $\mathcal{T}$, where $\Sigma_{3} \wedge \Pi_{3} \vdash F_{4}$ is not derived by the Fig. 14: Transformed proof tree $\mathcal{T}^{\prime}$ induction rule Ind

By modifying the rule $\mathrm{AH}$, we can transform the proof tree $\mathcal{T}$ into a proof tree $\mathcal{T}^{\prime}$, where every node of the tree $\mathcal{T}$ which applies a hypothesis (not derived by the rule Ind) to prove an entailment is replaced by a new node that contains not only the target entailment, but also full proof tree of the applied hypothesis. Since this transformation is performed only on hypotheses not derived by the rule Ind, it follows that in the new proof tree $\mathcal{T}^{\prime}$, only induction hypotheses recorded by the rule Ind participating into the mutual induction proof. We have shown earlier in the discussion about the rule $\mathrm{AH}$ that its side condition about model decreasing maps to the well-founded relation of our mutual induction principle. Therefore, the mutual induction principle is ensured by this new proof tree. This implies that all induction hypotheses (recorded by the rule Ind) are valid. Therefore, the original entailment is also valid. 